\title{
Diseño y validación de una escala de actitudes hacia la violencia de pareja para adultos colombianos $^{3}$
}

\author{
Doris Adriana Suárez Hernández \\ Psicóloga en formación \\ Universidad de La Sabana. Bogotá, Colombia \\ Correo electrónico: adrianasuarezhernandez.rs.d@gmail.com
}

Karen Julieth Riaño Maldonado

Psicóloga en formación

Universidad de La Sabana. Bogotá, Colombia

Correo electrónico: karenm9808@gmail.com

Leading Stefany Nova Bolivar Psicóloga en formación Universidad de La Sabana. Bogotá, Colombia Correo electrónico: este.fanynova97@gmail.com

Fernando Riveros Munévar

Magíster en Psicología

Universidad de La Sabana. Bogotá, Colombia Correo electrónico: edgar.riveros@unisabana.edu.co

Recibido: 03/12/2018

Evaluado: $15 / 01 / 2019$

Aceptado: $14 / 05 / 2019$

\section{Resumen}

El objetivo de este estudio fue diseñar y validar una escala psicométrica para medir las actitudes hacia la violencia de pareja. Para ello se realizó una revisión teórica, derivando a una tabla de especificaciones que guió la construcción de los reactivos, seguido de un proceso de evaluación por jueces, la depuración de los reactivos y ajuste de la escala para la posterior aplicación a 357 personas, para luego llevar a cabo el análisis factorial de los datos obtenidos, y un análisis de confiabilidad y consistencia interna. Como resultado se construyó un instrumento conformado por 30 reactivos, con altos índices de validez (> .6) y confiabilidad (.859) para medir actitudes hacia la violencia de pareja.

Palabras clave

Actitudes, violencia de pareja, psicometría.

3 Para citar este artículo: Suárez, D.A., Riaño, K.J., Nova, L.S. \& Riveros, F. (2020). Diseño y validación de una escala de actitudes hacia la violencia de pareja para adultos colombianos. Informes Psicológicos, 20(1), pp. 33-47 http:// dx.doi.org/10.18566/infpsic.v20n1a03 


\title{
Design and validation of a scale of attitudes towards intimate partner violence for Colombian adults
}

\begin{abstract}
The purpose of this study was to design and validate a psychometric scale to measure attitudes towards intimate partner violence. To do so, a theoretical review was started, and then a specification table was designed, which guided the construction of the reagents. Then, the process of evaluation by judges, the purification of the reagents and the adjustment of the scale for its subsequent application to 357 people were carried out. Finally, the factor analysis of the data obtained was made, as well as an analysis of reliability and internal consistency. As a result, an instrument consisting of 30 reagents was built, with high rates of validity $(>.6)$ and reliability $(.859)$ to measure attitudes towards intimate partner violence.
\end{abstract}

Keywords Attitudes, intimate partner violence, psychometrics.

\section{Projeto e validação de uma escala de atitudes em relação à violência no casal para adultos colombianos}

\section{Resumo}

0 objetivo desde estudo foi projetar e validar uma escala psicométrica para medir as atitudes em relação à violência no casal. Com esse fim foi feita uma revisão teórica, decorrendo em uma tábua de especificações que norteio a construção dos reativos, seguido de um processo de avaliação por juízes, a depuração dos reativos e 0 ajuste da escala para posterior aplicação a 357 pessoas, e posteriormente fazer a análise fatorial dos dados obtidos, e uma análise de confiabilidade e consistência interna. Como resultado construiu-se um instrumento conformado por 30 reativos, com altos índices de validade (> .6) e confiabilidade (.859) para medir atitudes perante a violência no casal. 


\section{ntroducción}

Las relaciones interpersonales sanas son esenciales en la vida de los seres humanos, basándose en un beneficio mutuo que las conduce a un desarrollo integral, sin embargo, las relaciones que se establecen no siempre son saludables, ya que se puede presentar maltrato por parte de la pareja actual o precedente (Planed Parenthood Federation of America, 2005). La violencia de pareja es considerada como toda conducta que causa daño entre los individuos que mantienen una relación íntima como noviazgo, matrimonio o cohabitación (Grosman, 1992, citado en Almenares, Louro \& Ortiz, 1999), donde las víctimas o los victimarios pueden ser hombres, mujeres, jóvenes, ancianos, homosexuales, bisexuales o heterosexuales (Planed Parenthood Federation of America, 2005). Se manifiesta una posición de dominio y autoridad sobre la persona, cuando la intensidad y frecuencia aumentan debido a la repetición de la conducta (Jiménez, Barba, Fuentes, López \& Villacreces, 2015).

Este tipo de conducta violenta se puede evidenciar en una relación amorosa de tres formas: la primera es la violencia física, que comprende toda lesión física o corporal generada a partir de golpes, bofetadas, empujones y otros tipos de agresión los cuales causan marcas visibles; la segunda, comprende la violencia sexual refiriéndose a todos los actos sexuales realizados sin el consentimiento de la persona que es agredida, por último, la violencia psicológica, que es considerada como la destrucción verbal, gestual o conductual entre los sujetos, donde se manifiestan insultos, críticas constantes, humillaciones y descalificación continua
(Grosman, 1992, citado en Almenares et al., 1999).

La violencia de pareja tiene una alta prevalencia en Colombia, ya que se reportaron 527.284 casos entre el año 2007 y el 2016, en este último año con un aumento del $7 \%$, con una tasa de 126,30 por cada 100.000 personas y una prevalencia mayor en el rango de edad entre los 25 y los 29 años (Medicina Legal y Ciencias Forenses, 2017).

La violencia ha sido abordada desde múltiples autores quienes han hecho diversos estudios. Algunos de ellos han investigado las diferencias entre la violencia ejercida por hombres y mujeres; donde se evidencian diferencias significativas entre hombres y mujeres al ejercer violencia sexual, ya que se identifica que las mujeres han sido más agredidas que los hombres, mientras que, en la violencia física y psicológica, se presentan cifras similares en los reportes de hombres y mujeres. Así mismo, se encuentra que la violencia física y la agresión verbal han tenido el mayor número de reportes (Benavides, 2016).

Adicionalmente, se han estudiado las actitudes hacia la violencia social, como por ejemplo Ramírez, Quintero, Aguilar y Méndez (2007), quienes evaluaron actitudes de venganza, disfrute vengativo, imposición abusiva, evitación o aceptación de la agresión entre iguales, encontrando que la violencia es usada como un mecanismo de justicia, sin embargo es rechazada cuando los sujetos de agresión son ellos mismos y que esas actitudes hacia la violencia se relacionan con el porcentaje de riñas en los estudiantes de Bucaramanga. 
Por otro lado, la violencia física, sexual y psicológica ha sido investigada con el propósito de identificar la intensidad y la frecuencia de la violencia doméstica y los casos de hombres y mujeres que son denunciados por este tipo de conductas. Esto se relaciona con el deterioro de la relación y el grado de violencia. Las cifras de denuncias de violencia en hombres y mujeres superan los baremos; a pesar de esto, las parejas se mantienen en la relación esperando cambios por parte de su compañero sentimental (Carrasco, 2004). Por su parte, estudios como el de Benavides (2016) muestran cómo la violencia de pareja se presenta desde el mismo noviazgo, en tempranos momentos y cómo tanto hombres como mujeres son víctimas de la misma.

También se han hecho investigaciones sobre las creencias y actitudes hacia la violencia de pareja contra la mujer, evidenciando que las actitudes tolerantes hacia este tipo de conductas son un factor de riesgo para la presencia de la violencia de pareja. Otros factores de riesgo son las creencias hacia el rol de género (hay una aceptación sobre los roles tradicionales que desempeñan hombres y mujeres como la subordinación femenina y la dominancia masculina) y el nivel académico (las personas con una menor educación o sin una formación sobre este tema son más tolerantes y tienen un menor juicio hacia la violencia), sin embargo, el nivel educativo no garantiza que no se presenten actitudes favorables hacia la violencia de pareja contra la mujer (Ferrer, Bosch, Ramis, Torres \& Navarro, 2006).

Las actitudes son consideradas como una disposición intrínseca que es aprendida y tiende a permanecer en el tiempo. Se da como una respuesta de aceptación o rechazo hacia ciertos tipos de objetos, ideas o personas ubicadas en el mundo social. Este proceso se estima como una evaluación que se crea a partir de los juicios y valores que son influidos por las experiencias que ha tenido el individuo con los objetos, las personas y con determinadas ideas (León et al., 1998). Además de esto, las creencias, sentimientos o la intención de su conducta, ya sean individuales o colectivas, también influye en las actitudes (Myers, 2005; Abello et al., 2018).

Las actitudes son producto de la adaptación, ya que tienen la intención de mejorar las relaciones interpersonales para disminuir la probabilidad de obtener un castigo, por lo que pueden ser modificadas a medida que el conocimiento y la interpretación de la realidad del entorno se amplía y adquiere otro significado (Sabucedo \& Morales, 2015). Si el entorno social en el que está inmerso el agresor no valora como negativa la conducta agresiva y si su pareja no reporta la primera vez que ocurre la agresión, la persona no va a modificar la interpretación que tiene sobre la violencia y su práctica. Cada día se reciben en el Instituto Nacional de Medicina Legal 140 casos donde las víctimas reportan la violencia ejercida por su pareja cuando es lo suficientemente grave para buscar atención y no en el momento que comenzó este tipo de agresiones, lo cual dificulta la identificación y modificación de estos casos, ya que, si bien, esperan a que la agresión se repita varias veces, cuando deciden hacer la denuncia abandonan los casos, lo que da paso a la impunidad (Manjarrés, 2017).

Este tipo de actitudes pueden ser explicadas desde la división tridimensional que hace León et al. (1998) desde su 
significado social: el primero de estos es el individual, donde el medio social le da modelos y apoyos; el segundo es interpersonal, construido como base de atracción mutua y formación de grupos y, por último, el colectivo, donde se observan semejanzas y diferencias individuales en el núcleo de la sociedad.

Es importante tener en cuenta que las actitudes contienen aspectos tales como el cognitivo, el afectivo y el conductual.

El primer componente de las actitudes hace énfasis en los atributos, juicios o creencias que se le asignan a una persona, objeto o grupo de individuos, mientras que el segundo expone las emociones o sentimientos que se manifiestan hacia el individuo, el objeto o hacia un grupo establecido. Finalmente, el último factor se refiere a las conductas ya ocurridas que se tienen sobre el objeto, el sujeto o sobre cierto grupo (Abello et al., 2018; Maio \& Haddock, 2014; Riveros, Bohórquez, López \& Sepúlveda, 2015). Es decir, se establece una relación entre lo que la persona siente, piensa, hace y cómo lo manifiesta en el ambiente privado y público (Myers, 2005).

Ante la violencia de pareja, las personas toman una postura donde evalúan la situación que se está presentando en el país, en la cual se asume una posición a favor o en contra hacia este tipo de conductas. Esto es importante, ya que las actitudes pueden influir en las decisiones que toman y las acciones que realizan las personas (Sabucedo \& Morales, 2015), lo que es relevante frente a la violencia de pareja, ya que la evaluación de las actitudes de las personas hacia este tipo de comportamiento permitiría observar cómo la sociedad percibe la violencia.
En América Latina la violencia es considerada como el producto de pactos patriarcales, ya que en la mayoría de ocasiones las víctimas que reportan estos delitos son mujeres. Esto es visto como un fenómeno cultural que condiciona el comportamiento de las personas, lo que requiere campañas preventivas y cambios que eviten este tipo de actos violentos (Hurtado \& Fríes, 2010). La violencia de pareja es determinada por conceptos sexistas, donde se ve involucrado un componente cognitivo, características biológicas de hombres y mujeres, y un componente afectivo, que tiene que ver con la construcción de identidades en base al rol que ejerce desde su sexo (Díaz-Agudelo, 2001, citado en Vélez, 2015). Adicionalmente, la necesidad de evaluar la actitud hacia la violencia de pareja se sostiene debido a que las actitudes de las personas intervienen en sus relaciones sentimentales, donde influyen aspectos como la expectativa que se tiene de la relación y cómo la persona debe comportarse en la intimidad (Ferrer, Esperanza \& Ramis, 2006).

Este tipo de violencia basado en el sexo de la persona y dirigido por su pareja se ha evaluado en escalas como "Actitud hacia la violencia contra la mujer en la relación de pareja y el clima social familiar en adolescentes" que está validada con muestras de Lima (Pacheco, 2015), y otras donde el sexo no es relevante, como la "Acceptance of couple violence" (Dahlberg, Toal, \& Behrens, 1998), el "The Attitudes Towards Dating Violence Scales" (Price et al., 1999), o el "Intimate Partner Violence Attitude Scale", validado con personas estadounidenses (Fincham, Cui, Braithwaite \& Pasley, 2008); sin embargo no existen escalas validadas en la población Colombiana, y 
las existentes no se ajustan al contexto ni al lenguaje colombiano, lo que hace relevante la creación de una escala que mida las actitudes hacia la violencia de pareja en su contexto.

En conclusión, la violencia de pareja es una problemática concurrente en la consulta clínica lo que sustenta su medición al respecto, ya existen escalas diseñadas para ello pero, no se ajustan al contexto colombiano, por esta razón, la presente investigación considera importante diseñar y validar una prueba que mida las actitudes hacia la violencia de pareja, reflejando los componentes de la actitud de la persona (cognitivo, afectivo y conductual), y los tipos de violencia ejercida (sexual, física y psicológica).

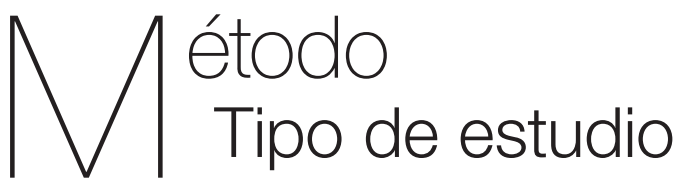

La presente investigación tiene como objetivo el diseño y la validación de la escala de actitudes hacia la violencia de pareja, por lo que se considera como un estudio instrumental. Este tipo de estudio está dirigido a la construcción de pruebas, donde se incluye su diseño y las características psicométricas (Montero \& León, 2005).

\section{Participantes}

Participaron 357 estudiantes universitarios de la Sabana de Bogotá y los municipios cercanos quienes habían estado en una relación de pareja con anterioridad, de los cuales 221 (61.2\%) fueron mujeres y 136 (37.7\%) hombres, con un rango de edad entre los 18 y los 30 años
$(\mathrm{M}=20.2$ y $\mathrm{S}=2.48)$; la muestra utilizada fue obtenida por un muestreo no probabilístico a conveniencia.

\section{Procedimiento}

Después de realizar la revisión teórica, se construyó la tabla de especificaciones asignando los componentes de la actitud (cognitivo, conductual, afectivo) y la violencia (física, psicológica y sexual) para la creación de los reactivos. Posterior al diseño de la escala, se presentó a los jueces expertos para la validación de cada uno de los ítems. Para la validación por jueces se hizo una invitación formal, y luego de aceptar su participación como jueces, se les entregó el formato de evaluación, los ítems que componían la escala, una breve revisión teórica y la tabla de especificaciones. Dadas las recomendaciones de los jueces, se realizaron correcciones a diferentes ítems y, así mismo, se eliminaron los que no se consideraban pertinentes para medir las actitudes hacia a la violencia de pareja. Luego, se procedió a la aplicación de la escala, presentando un consentimiento informado a los participantes y, seguido de éste, se les entregó la escala que debían contestar.

Para el análisis de datos se utilizó SPSS versión 24, comprobando si el tamaño muestral era adecuado, haciendo uso de herramientas estadísticas como la medida de Kaiser-Meyer-Olkin y, posteriormente, realizar el análisis factorial exploratorio por método de máxima verosimilitud y rotación oblimin para identificar la validez de constructo, además del análisis de consistencia interna a través del Alfa de Cronbach y el de confiabilidad de dos mitades, así como correlaciones 
elemento-total corregido y correlaciones entre elementos.

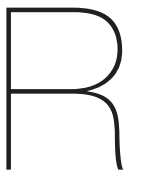

\section{esultados}

A continuación, se presenta la tabla de distribución inicial de reactivos (Tabla 1) según los componentes que se derivan de la actitud (cognitivo, afectivo y conductual) y de la violencia (sexual, física y psicológica).

Tabla 1.

Distribución inicial de los reactivos

\begin{tabular}{|c|c|c|}
\hline $\mathrm{N}^{\circ}$ de ítems & Conceptos & Componentes \\
\hline $\begin{array}{l}\text { (8) Numerales. } \\
50,63,31,39 \\
26,59,18,37\end{array}$ & $\begin{array}{l}\text { Creencias, características o } \\
\text { juicios dados sobre las lesiones } \\
\text { físicas causadas a un individuo. }\end{array}$ & $\begin{array}{l}\text { Cognitivo-V. } \\
\text { física }\end{array}$ \\
\hline $\begin{array}{c}\text { (8) Numerales. } \\
68,51,25,46 \\
9,33,15,3\end{array}$ & $\begin{array}{l}\text { Manifestación de emociones } \\
\text { o sentimientos que se expre- } \\
\text { san hacia las lesiones físicas } \\
\text { causadas a un individuo. }\end{array}$ & $\begin{array}{l}\text { Afectivo-V. } \\
\text { física }\end{array}$ \\
\hline $\begin{array}{l}\text { (9) Numerales. } \\
42,23,17 \\
28,66,58 \\
52,41,36\end{array}$ & $\begin{array}{l}\text { Acciones ejercidas frente } \\
\text { a las lesiones físicas cau- } \\
\text { sadas a un individuo. }\end{array}$ & $\begin{array}{l}\text { Conductu- } \\
\text { al-V. física }\end{array}$ \\
\hline $\begin{array}{l}\text { (5) Nume- } \\
\text { rales. 29, 8, } \\
14,13,60 \text {. }\end{array}$ & $\begin{array}{l}\text { Creencias, características o } \\
\text { juicios dados a los sucesos } \\
\text { sexuales ejecutados sin la } \\
\text { aprobación del individuo. }\end{array}$ & Cognitivo-sexual \\
\hline $\begin{array}{l}\text { (5) Numerales. } \\
47,6,61,4,2\end{array}$ & $\begin{array}{l}\text { Manifestación de emociones o } \\
\text { sentimientos que se expresan hacia } \\
\text { los sucesos sexuales ejecutados } \\
\text { sin la aprobación del individuo. }\end{array}$ & Afectivo-sexual \\
\hline $\begin{array}{l}\text { (6) Numerales. } \\
54,67,53 \\
49,38,5\end{array}$ & $\begin{array}{l}\text { Acciones ejercidas frente a los } \\
\text { sucesos sexuales realizados sin } \\
\text { la aprobación del individuo }\end{array}$ & $\begin{array}{l}\text { Conductu- } \\
\text { al-V. sexual }\end{array}$ \\
\hline $\begin{array}{c}\text { (12) Nume- } \\
\text { rales.30, 32, } \\
64,56,35 \\
20,22,27,11 \\
55,43,16\end{array}$ & $\begin{array}{l}\text { Creencias, características o } \\
\text { juicios dados sobre la destruc- } \\
\text { ción verbal, gestual o conduc- } \\
\text { tualmente de un individuo. }\end{array}$ & $\begin{array}{l}\text { Cognitivo-psi- } \\
\text { cológico }\end{array}$ \\
\hline $\begin{array}{l}\text { (10) Numerales. } \\
69,45,10,73 \\
72,21,57 \\
19,71,62\end{array}$ & $\begin{array}{l}\text { Manifestación de emociones o } \\
\text { sentimientos que se expresan hacia } \\
\text { la destrucción verbal, gestual o } \\
\text { conductualmente de un individuo }\end{array}$ & $\begin{array}{l}\text { Afectivo-psi- } \\
\text { cológico }\end{array}$ \\
\hline $\begin{array}{l}\text { (10) Numerales. } \\
24,44,48 \\
12,65,40 \\
70,7,1,34\end{array}$ & $\begin{array}{l}\text { Acciones ejercidas frente a } \\
\text { la destrucción verbal, gestual } \\
\text { o.conductualmente de un individuo. }\end{array}$ & $\begin{array}{l}\text { Conductual-V. } \\
\text { psicológico }\end{array}$ \\
\hline
\end{tabular}

A partir de la distribución de los reactivos y su construcción, se realizó la validación de jueces según las calificaciones asignadas a los ítems, en base a la modificación del modelo de Lawshe de los acuerdos necesarios para la conservación de los ítems (Tristán, 2008), en términos de la pertinencia, la suficiencia, la coherencia y la redacción de los ítems con respecto a la calificación que los seis jueces les asignaron (2 psicómetras y cuatro profesionales que trabajan con el tema de pareja y violencia).

Teniendo en cuenta los criterios que propone Tristán (2008) según el modelo de Lawshe se conservan 51 ítems, ya que su coeficiente de validez de contenido está por encima de .67 lo que es necesario para que el ítem sea aceptado según el número de jueces que participaron. Por lo se conservan 51 ítems de 73. Los demás ítems fueron eliminados, debido a que no cumplieron con los criterios de pertinencia, suficiencia o no fueron aceptables en tres de los criterios de conservación $(<.67)$.

Después de la aplicación de la escala con estudiantes universitarios, se examinó la conveniencia del tamaño muestral a través, de los estadísticos de KaiserMeyer-Olkin y la prueba de esfericidad de Bartlett. Los datos demuestran que la muestra que participó en la validación de la escala fue suficiente con una medida de KMO de .88 y una significancia de Bartlett de .000, lo que permite realizar el análisis factorial. Los datos de extracción muestran que todos los reactivos de la escala tienen una extracción adecuada (> .3), lo que significa que todos los ítems pueden mantenerse. Posterior a esto, se establecen la cantidad de factores que pertenecen a la escala como se muestra 
en la Tabla 2. A través del análisis factorial exploratorio, la regla de Kaiser de autovalores superiores a 1 y el análisis paralelo de Horn, se determina el número de factores, así como la congruencia con la tabla de especificaciones y los ítems que la componen.

Tabla 2.

Extracción de factores tras el análisis de componentes principales

\begin{tabular}{ccc}
\hline \multicolumn{3}{c}{ Varianza total explicada } \\
\hline Componente & Sumas de rotación de cargas al cuadrado \\
\hline 1 & Total & \% acumulado \\
\hline 2 & 5.36 & 17.867 \\
3 & 2.608 & 26.561 \\
4 & 2.124 & 33.64 \\
5 & 1.966 & 40.195 \\
6 & 1.96 & 46.727 \\
7 & 1.725 & 52.477 \\
\hline
\end{tabular}

Como muestran los resultados de la Tabla 2, la escala está compuesta por 7 factores que explican en un 57.24\% la varianza acumulada. Después de obtener el número de factores que componen la escala, se determinaron los reactivos que hacen parte de cada factor (Tabla 3).

En la Tabla 3 se evidencia cómo está constituido cada factor según sus reactivos. Se evidencia que el primer factor nombrado como conductual psicológico se constituye por los reactivos 20, 22, 24, 25, 26, 34, 38, 39, 40 y 45. Así mismo, el segundo factor conductual físico se conforma por los reactivos $12,17,28,36$, 47; luego, el tercer factor se denomina como cognitivo sexual y se compone por los reactivos 8,9 y 13. De la misma forma, el cuarto factor fue llamado como cognitivo psicológico, constituyéndose
Tabla 3

Reactivos y su correspondiente factor.

\begin{tabular}{|c|c|c|c|c|c|c|c|}
\hline \multicolumn{8}{|c|}{ Matriz de componente rotados } \\
\hline & \multicolumn{7}{|c|}{ Componente } \\
\hline & 1 & 2 & 3 & 4 & 5 & 6 & 7 \\
\hline Ítem 20 & .736 & & & & & & \\
\hline Ítem 22 & .653 & & & & & & \\
\hline Ítem 24 & .737 & & & & & & \\
\hline Ítem 25 & .499 & & & & & & \\
\hline Ítem 26 & .826 & & & & & & \\
\hline Ítem 34 & .704 & & & & & & \\
\hline Ítem 38 & .587 & & & & & & \\
\hline Ítem 39 & .595 & & & & & & \\
\hline Ítem 40 & .716 & & & & & & \\
\hline Ítem 45 & .744 & & & & & & \\
\hline Ítem 12 & & .558 & & & & & \\
\hline Ítem 17 & & .723 & & & & & \\
\hline Ítem 28 & & .545 & & & & & \\
\hline Ítem 36 & & .793 & & & & & \\
\hline Ítem 47 & & .673 & & & & & \\
\hline Ítem 1 & & & & & & & .727 \\
\hline Ítem 3 & & & & & & & .67 \\
\hline Ítem 50 & & & & & & & .432 \\
\hline Ítem 8 & & & .74 & & & & \\
\hline Ítem 9 & & & .716 & & & & \\
\hline Ítem 13 & & & .675 & & & & \\
\hline Ítem 10 & & & & & & .731 & \\
\hline Ítem 14 & & & & & & .713 & \\
\hline Ítem 15 & & & & & & .639 & \\
\hline Ítem 32 & & & & .736 & & & \\
\hline Ítem 33 & & & & .764 & & & \\
\hline Ítem 49 & & & & .657 & & & \\
\hline Ítem 35 & & & & & .599 & & \\
\hline Ítem 42 & & & & & .77 & & \\
\hline Ítem 43 & & & & & 681 & & \\
\hline
\end{tabular}

por los reactivos 35, 42 y 43; mientras que el quinto factor, nombrado como afectivo físico, se forma por los reactivos 10, 14 y 
15. El sexto factor, denominado cognitivo físico, se conforma por los reactivos 1, 3 y 50; y el último factor se nombró como conductual sexual y se constituye por los reactivos 35,42 y 43 .

Para finalizar, los datos demostraron una confiabilidad de .859, según el Alfa de Cronbach; así mismo, se usaron estadísticos para el análisis de consistencia interna según las correlaciones inter-elementos y análisis de correlaciones entre elemento-total corregida donde todos los ítems presentan correlaciones positivas y superiores a .3 entre los reactivos y correlaciones significativas y superiores a .4 entre cada ítem y el total de la prueba.

Con la eliminación de los ítems, según la validación por jueces, los análisis factoriales y la consistencia interna, quedaron 30 ítems, en los siete factores, conductual psicológico, cognitivo psicológico, conductual físico, cognitivo físico, afectivo físico, cognitivo sexual y conductual sexual, como se muestra en la Tabla 4.

Tabla 4.

Tabla final de especificaciones.

\begin{tabular}{|c|c|c|}
\hline $\mathrm{N}^{\circ}$ de ítems & Conceptos & $\begin{array}{l}\text { Compo- } \\
\text { nentes que } \\
\text { conforman } \\
\text { la actitud }\end{array}$ \\
\hline $\begin{array}{c}\text { (3) Numerales } \\
1,2,30\end{array}$ & $\begin{array}{l}\text { Creencias, característi- } \\
\text { cas o juicios dados } \\
\text { sobre las lesiones físicas } \\
\text { causadas a un individuo. }\end{array}$ & $\begin{array}{l}\text { Cognitivo-V. } \\
\text { física }\end{array}$ \\
\hline $\begin{array}{l}\text { (3) Numera- } \\
\text { les } 5,8,9\end{array}$ & $\begin{array}{l}\text { Manifestación de } \\
\text { emociones o sentimien- } \\
\text { tos que se expresan } \\
\text { hacia las lesiones físicas } \\
\text { causadas a un individuo. }\end{array}$ & $\begin{array}{l}\text { Afectivo-V. } \\
\text { física }\end{array}$ \\
\hline $\begin{array}{c}\text { (5) Numerales } 6 \text {, } \\
10,16,21,28\end{array}$ & $\begin{array}{l}\text { Acciones ejercidas } \\
\text { frente a las lesiones } \\
\text { físicas causadas } \\
\text { a un individuo. }\end{array}$ & $\begin{array}{l}\text { Conductu- } \\
\text { al-V. física }\end{array}$ \\
\hline $\begin{array}{l}\text { (3) Numera- } \\
\text { les } 3,4,7\end{array}$ & $\begin{array}{l}\text { Creencias, característi- } \\
\text { cas o juicios dados a } \\
\text { los sucesos sexuales } \\
\text { ejecutados sin la } \\
\text { aprobación del individuo. }\end{array}$ & $\begin{array}{c}\text { Cogniti- } \\
\text { vo-sexual }\end{array}$ \\
\hline
\end{tabular}

Continuación

\begin{tabular}{|c|c|c|}
\hline $\mathrm{N}^{\circ}$ de ítems & Conceptos & $\begin{array}{l}\text { Compo- } \\
\text { nentes que } \\
\text { conforman } \\
\text { la actitud }\end{array}$ \\
\hline $\begin{array}{c}\text { (3) Numerales } \\
20,25,26\end{array}$ & $\begin{array}{l}\text { Acciones ejercidas } \\
\text { frente a los sucesos sex- } \\
\text { uales realizados sin la } \\
\text { aprobación del individuo. }\end{array}$ & $\begin{array}{l}\text { Conductu- } \\
\text { al-V. sexual }\end{array}$ \\
\hline $\begin{array}{c}\text { (3) Numerales } \\
20,25,26\end{array}$ & $\begin{array}{l}\text { Creencias, característi- } \\
\text { cas o juicios dados } \\
\text { sobre la destrucción } \\
\text { verbal, gestual o } \\
\text { conductualmente } \\
\text { de un individuo. }\end{array}$ & $\begin{array}{l}\text { Cognitivo-psi- } \\
\text { cológico }\end{array}$ \\
\hline $\begin{array}{c}\text { (10) Numerales } \\
11,12,13 \\
14,15,19,22 \\
23,24,27\end{array}$ & $\begin{array}{l}\text { Acciones ejercidas } \\
\text { frente a la destruc- } \\
\text { ción verbal, gestual } \\
\text { 0.conductualmente } \\
\text { de un individuo. }\end{array}$ & $\begin{array}{l}\text { Conductual-V. } \\
\text { psicológico }\end{array}$ \\
\hline
\end{tabular}

$\int$ ISCUSIÓn dio fue diseñar y validar una escala de actitudes hacia la violencia de pareja, ya que en el contexto colombiano es relevante estudiar este fenómeno, que se ha incrementado un 6\% desde año 2013 hasta el 2016 (Manjarrés, 2016). El estudio de las actitudes hacia la violencia de pareja aportaría a entender el fenómeno, ya que pueden ayudar al mantenimiento o no de esta conducta en los agresores (Sabucedo \& Morales, 2015).

En la escala se determinaron distintos factores: el conductual psicológico responde a las acciones que ejerce la persona frente a una situación que muestre destrucción verbal o gestual hacia un individuo; luego el conductual físico se relaciona con las acciones ejercidas de un sujeto al ver que una persona causa las lesiones físicas a un individuo; por otro lado, el cognitivo sexual plantea los pensamientos o juicios que tiene la persona frente a los sucesos sexuales ejecutados 
sin la aprobación de algún individuo; el cognitivo psicológico, se refiere a las creencias o pensamientos del sujeto respecto a una situación donde haya destrucción verbal o gestual hacia un individuo; adicionalmente, el afectivo físico plantea la manifestación de emociones o sentimientos que se experimentan y expresan al observar que se ejecutan lesiones físicas hacia un individuo; el cognitivo físico tiene en cuenta las creencias o pensamientos que tiene la persona respecto a lesiones físicas causadas a un individuo; por último, el factor conductual sexual, establece la disposición que tendría una persona de ejercer conductas frente al tener conocimiento de actos sexuales realizados sin la aprobación de un individuo.

Los resultados encontrados en esta investigación muestran una escala compuesta por siete factores que explican el $57.24 \%$ de la varianza acumulada, con una adecuada coherencia teórica con los reactivos, además de altos indicadores de confiabilidad y consistencia interna. En la escala final quedaron 30 ítems (Anexo 1), con las correspondientes correcciones e ítems que debían ser eliminados y los percentiles en el Anexo 2.

Algunas limitaciones del presente estudio son el tamaño de la muestra, ya que, aunque con un buen índice en la medida de $\mathrm{KMO}$, pudo haber utilizado un tamaño muestral mayor. La representatividad de la muestra también es un problema, ya que solo participaron personas de Bogotá y pueblos aledaños, y, aunque Bogotá es uno de los lugares con mayor incidencia de casos denunciados de violencia, también es importante aplicar la prueba con personas de otros departamentos. Los participantes, en su gran mayoría, eran estudiantes universitarios, y el nivel de educación puede ser considerado como un factor importante al momento de evaluar la aceptación o el rechazo hacia la violencia de pareja, ya que se podrían encontrar diferencias. Por ello, en futuros estudios se recomienda utilizar una muestra con un mayor tamaño muestral y con una mayor representatividad.

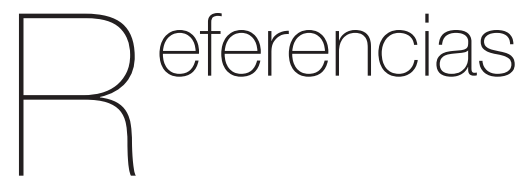

Abello, E., Bello, J., Güiza, A., Ortiz, A. M., Riveros, F., Vera, L.A., Bernal, A.P., \& Reyes, L. (2018). Actitudes hacia los niños adoptables por parte de familias monoparentales y nucleares de estratos 4, 5 y 6 en la ciudad de Bogotá. Informes Psicológicos, 18(2), 45-62. http://dx.doi. org/10.18566/infpsic.v18n2a03

Almenares, M., Louro, I., \& Ortiz, M. (1999). Comportamiento de la violencia intrafamiliar. Revista Cubana de Medicina General Integral, 15, 285-292.

Benavides, J. (2016). Violencia en el noviazgo: Diferencias de Género. Informes Psicológicos, 16(2), 27-36. http://dx.doi. org/10.18566/infpsicv16n2a02

Carrasco, J. C. (2004). Violencia física, psicológica y sexual en el ámbito de la pareja: papel del contexto. Clínica y salud: Revista de Psicología Clínica y Salud, 15(1), 33-54.

Dahlberg, L. L., Toal, S. B., \& Behrens, C. B. (Eds.). (1998). Measuring violence-related attitudes, beliefs, and behaviors among youths: A compendium of assessment 
tools. Atlanta, GA: Division of Violence Prevention, National Center for Injury Prevention and Control, Centers for Disease Control and Prevention.

Ferrer, V. A., Bosch, E., Ramis, M. C., Torres, G., \& Navarro, C. (2006). La violencia contra las mujeres en la pareja: creencias y actitudes en estudiantes universitarios/as. Psicothema, 18(3), 359-366.

Ferrer, V., A., Esperanza, B. F., \& Ramis Palmer, M. C. (2006). Las creencias y actitudes sobre la violencia contra las mujeres en la pareja: determinantes sociodemográficos, familiares y formativos. Anales de Psicología, 22(2), 251-259.

Fincham, F.D., Cui, M., Braithwaite, S.R., \& Pasley, K. (2008). Attitudes towards intimate partner violence in dating relationships. Psychological Assessment, 20, 260-269.

Hurtado, V., \& Fríes, L. (2010). Estudio de la información sobre la violencia contra la mujer en América Latina y el Caribe. Santiago de Chile: Cepal.

Jiménez, A., Barba, M., Fuentes, M., López, E., \& Villacreces, N. (2015). Violencia de la mujer hacia el hombre, ¿mito o realidad? Reidocrea: Revista Electrónica de Investigación y Docencia Creativa, 4, 14-17.

León, J., Barriga, S., Gómez, T., González, B., Medina, S., \& Cantero, F., (1998). Psicología social: Orientaciones teóricas y ejercicios prácticos. (1st. ed.). Madrid: McGraw-Hill/ Interamericana de España.

Maio, G. \& Haddock, G. (2014). The Psychology of Attitudes and Attitude Change (2nd ed.). London: Sage.
Manjarrés, A., (2017). Cada día, 140 mujeres son agredidas por sus parejas en Colombia. Instituto Nacional de Medicina Legal. Recuperado de http://www.medicinalegal. gov.co/cada-dia-140-mujeres-sonagredidas-por-sus-parejas-en-colombia

Montero, I. \& León, O. (2005) Sistema de clasificación del método en los informes de investigación en Psicología. International Journal of Clinical and Health Psychology, 5(1), 115-127. Recuperado de http://www. redalyc.org/articulo.oa?id=33701007

Myers, D., (2005). Psicología social (8a ed.). México: McGraw Hill - Interamericana.

Pacheco, M. (2015). Actitud hacia la violencia contra la mujer en la relación de pareja y el clima social familiar en adolescentes. Interacciones. Revista de Avances en Psicología, 1(1), 29-44. http://dx.doi. org/10.24016/2015.v1n1.2

Planed Parenthood Federation of America. (2005). Elmaltrato en las relaciones, la violencia con la pareja íntima y la violencia doméstica constituyen una amenaza para las personas y para la sociedad. Recuperado de https://www. plannedparenthood.org/files/7313/9980/7759/ EMMaltratoEnLasRelacionesLaViolencia_06-05. pdf

Price, E., Byers, E., Belliveau, N., Bonner, R., Caron, B., \& Doiron, D. et al. (1999). The Attitudes Towards Dating Violence Scales: Development and Initial Validation. Journal of Family Violence, 14(4), 351-375. http:// dx.doi.org/10.1023/a:1022830114772

Ramírez, L. Y. Á., Quintero, A. P. C., Aguilar, P. F., \& Méndez, S. V. (2007). Actitudes hacia la violencia social entre iguales y su relación con variables sociodemográficas en tres grupos de estudiantes de secundaria 
de la ciudad de Bucaramanga. Revista Colombiana de Psicología, 16, 127-137.

Riveros, F., Bohórquez, D., López, S. \& Sepúlveda, E. (2015). Diseño y validación de un instrumento para medir las actitudes frente a la labor profesional del psicólogo. Revista Iberoamericana de Psicología: Ciencia y Tecnología, 8(2), 55-66.

Sabucedo, J., \& Morales, J., (2015). Psicología social. Madrid: Editorial Médica Panamericana.

Tristán, A. (2008). Modificación al modelo de Lawshe para el dictamen cuantitativo de la validez de contenido de un instrumento objetivo. Avances en Medición, 6(1), 37-48.

Vélez, M. A., (2015). Comportamiento de la Violencia de Pareja. Colombia, 2015. Instituto Nacional de Medicina Legal y Ciencias Forenses. Recuperado de http://www.medicinalegal.gov. co/documents/88730/3418907/5. VIOLENCIA DE PAREJA.pdf / 03de1fd1-092d-495f-99d3-1aacee2991c6 
$\bigwedge_{\text {Escala de Actitudes hacia la Violencia entre Pareja }}$

Nombre:

Edad:

\section{Instrucciones}

A continuación, encontrará una serie de afirmaciones. Lea cada una de ellas cuidadosamente y exprese su posición respecto a las siguientes afirmaciones, teniendo en cuenta estas opciones, totalmente desacuerdo (TD), desacuerdo (D), parcialmente desacuerdo (PD), parcialmente acuerdo (PA), acuerdo (A), totalmente acuerdo (TA).

No hay respuestas correctas o incorrectas, por lo que su sinceridad es importante.

\begin{tabular}{lllllll}
\hline ITEM & TD & D & PD & PA & A & TA \\
\hline 1. Me burlaría de mi pareja si siento que se ha equivocado & & & & & &
\end{tabular}

2. Me siento bien al ver que una persona cachetea a su pareja por infidelidad.

3. Está bien presionar o forzar a la pareja a tener relaciones sexuales.

4. Es obligación de una persona ceder ante los deseos sexuales de su pareja.

5. Me siento incómodo si veo que una persona golpea a su pareja.

6. Si tengo conocimiento de un caso de violencia de pareja, dejo que ellos lo solucionen porque una pareja es de dos, no de tres.

7. Controlar a la pareja por medio de la fuerza ayuda a que la relación se mantenga en orden.

8. Me molesta el hecho de que una persona utilice palabras groseras para dirigirse a su pareja en determinadas ocasiones.

9. NO está bien manipular a la pareja con hacerse daño a sí mismo

10. Si veo que alguien está siendo maltratado por su pareja, intervengo directamente diciéndole que no lo haga.

11. Esta bien el uso de fuerza si la pareja no obedece.

12. Es aceptable tener relaciones sexuales con su pareja por miedo a que esta le haga daño sino lo hace.

13. Le impediría a mi pareja tener contacto con su familia porque no me caen bien.

14. El dinero es un medio efectivo para ejercer el control sobre la pareja.

15. Si una persona quiere terminar la relación, está bien que agreda a su pareja 
16. Si escucho que alguien está siendo maltratado por su pareja, Ilamaría a la policía.

17. Me avergonzaría que mi pareja me pida hacer cosas que no quiero al momento de tener relaciones sexuales.

18. Me negaría si mi pareja me pide hacer cosas relacionadas con los actos sexuales en la cama que no quiero.

19. El uso de la fuerza puede ser empleado para corregir a la pareja cuando el caso lo amerite.

20. Me Siento impotente cuando veo que alguien está jaloneando a su pareja.

21. Intervendría si veo que una persona está agrediendo con golpes a su pareja.

22. Es normal que la persona sienta temor hacia su pareja.

23. Si una persona tiene razones para golpear a la pareja, me mantendría al margen.

24. Una persona puede pegarle a su pareja si lo ha ofendido.

25. Si sé de un caso de violencia sexual que no ha sido denunciado, me siento impotente.

26. Me irrita que una persona que es menos precia-

da por su pareja no haga nada al respecto.

27. Está bien amenazar a la pareja sino hace lo que se le dice.

28. Siento indiferencia hacia la violencia física de pare-

ja, porque eso solo les incumbe a ellos.

29. No permitiría que mi pareja se burle de mí en una reunión social

30. Siento remordimiento después de haber insultado a mi pareja 
$A^{\text {nexo } 2}$

\section{Tablas de calificaciones}

\begin{tabular}{cl}
\multicolumn{2}{c}{ Puntaje general } \\
\hline Puntaje & Interpretación \\
\hline $30-67$ & Actitud desfavorable \\
$68-105$ & Actitud con tendencia desfavorable \\
$106-143$ & Actitud con tendencia favorable \\
$144-180$ & Actitud favorable
\end{tabular}

\begin{tabular}{cl}
\hline \multicolumn{2}{c}{ Puntaje en violencia psicológica } \\
\hline Puntaje & Interpretación \\
\hline $13-28$ & Actitud desfavorable \\
$29-45$ & Actitud con tendencia desfavorable \\
$46-62$ & Actitud con tendencia favorable \\
$63-78$ & Actitud favorable \\
\hline
\end{tabular}

\begin{tabular}{cl}
\multicolumn{2}{c}{ Puntaje en violencia sexual } \\
\hline Puntaje & Interpretación \\
\hline $6-13$ & Actitud desfavorable \\
$14-21$ & Actitud con tendencia desfavorable \\
$22-29$ & Actitud con tendencia favorable \\
$29-36$ & Actitud favorable \\
\hline
\end{tabular}

Puntaje en violencia física

\begin{tabular}{cl}
\hline Puntaje & Interpretación \\
\hline $11-25$ & Actitud desfavorable \\
$26-39$ & Actitud con tendencia desfavorable \\
$40-53$ & Actitud con tendencia favorable \\
$54-66$ & Actitud favorable \\
\hline
\end{tabular}

